\title{
Sleep Related Expiratory Obstructive Apnea in Children
}

\author{
Mark E. Haupt, M.D. '; Denise M. Goodman, M.D., M.S.2; Stephen H. Sheldon, D.O., F.A.A.S.M. ${ }^{3}$ \\ ${ }^{1}$ Division of Pulmonary Medicine, ${ }^{2}$ Division of Critical Care Medicine, ${ }^{3}$ Division of Sleep Medicine, Ann \& Robert H. Lurie \\ Children's Hospital of Chicago, Northwestern University Feinberg School of Medicine, Chicago, IL
}

Study Objectives: We describe the respiratory, cardiac, and sleep-related characteristics of two types of sleep-related respiratory pauses in children that can fulfill current criteria of pathological apnea, but often seem to be benign: prolonged expiratory apnea (PEA) and post-sigh central apnea (PSCA). Methods: All outpatient comprehensive overnight polysomnography completed on children without significant underlying medical conditions completed during an 18-month period were retrospectively reviewed for the presence of augmented breaths followed by a respiratory pause. Events were identified as a PEA or PSCA based on characteristic features. Physiologic parameters associated with the respiratory events were recorded and compared.

Results: Fifty-seven (29 PEA and 28 PEA) events were identified in 17 patients $(8.5 \pm 3.5$ years old). Median durations of PEA and PSCA were not significantly different. For both PEA and PSCA, average heart rate $(\mathrm{HR})$ during the augmented breath before the respiratory pause differed from lowest instantaneous HR during the first half of the pause. When compared to each other, the lowest instantaneous HR recorded in the first half of PEA was lower than that for PSCA (63.9 [59.41-68.3] vs 66.75 [61.7-80.75]) beats per min, $p=0.03$. No PEA or PSCA event was associated with an oxygen desaturation more than $3 \%$ from baseline.

Conclusion: PEA and PSCA have stereotypic HR changes and resemble pathologic apneas but appear to be benign. Clinical significance of PEA and PSCA is yet to be determined. Consistent recognition of the events is required, given their frequency of occurrence and potential for misclassification.

Keywords: Sleep disorders, sleep apnea syndromes, pediatrics, apnea, polysomnography, exhalation

Citation: Haupt ME; Goodman DM; Sheldon SH. Sleep related expiratory obstructive apnea in children. J Clin Sleep Med 2012;8(6):673-679.
$\mathrm{P}$ rolonged apnea during infancy and childhood may be lifethreatening. Normal full-term infants and children, however, exhibit some respiratory pauses during sleep that are not pathological. ${ }^{1}$ Differentiation of normal physiological respiratory pauses and pathological apnea can be difficult.

Apnea is a transient cessation in respiration and can be classified as obstructive, central, or mixed. Obstructive apnea in children is conventionally defined as a $>90 \%$ decrease in airflow that lasts $\geq 90 \%$ of the duration of two normal breaths, as determined from the baseline breathing pattern, and has continued or increased respiratory effort during the period of decreased airflow. ${ }^{2}$ Central apnea occurs when inspiratory effort is absent during the entire period of airflow cessation. Current scoring rules require the event to last 20 seconds or to have at least two missed breaths and be associated with an electrocortical arousal, an awakening, or $\geq 3 \%$ desaturation. Mixed apnea occurs if there is absent inspiratory effort during the initial portion of the respiratory pause, followed by resumption of inspiratory effort before the end of the period of decreased airflow. Clearly, the focus of the definition of these events is related to the inspiratory phase oscillation of respiration.

Data do exist suggesting that significant respiratory events may occur only during the expiratory phase of the respiratory cycle in sleep. Southhall first presented the notion of prolonged expiratory apnea in children in 1985, demonstrating a relationship between upper airway obstruction and severe hypoxemia. ${ }^{3}$ Recent models of airflow limitation during the expiratory phase have been developed. ${ }^{4,5}$ Expiratory apneas are also described

\begin{abstract}
BRIEF SUMMARY
Current Knowledge/Study Rationale: Respiration during sleep in children is complex and differentiation of normal physiologic respiratory pauses and pathologic apneas can be difficult. The purpose of this study is to describe the respiratory, cardiac and sleep related characteristics of two types of sleep related respiratory pauses in children that can fulfill criteria for pathological apnea that, in this study, appear to be benign. Study Impact: We provide detailed description of two types of expiratory pauses in children: Prolonged Expiratory Apnea and Post-Sign Central Apnea. Consistent recognition and accurate scoring of such events is necessary given their frequency and potential for misclassification.
\end{abstract}

in children with Arnold-Chiari malformations, even in the absence of vocal cord paralysis or dysfunction. ${ }^{6,7}$ Woodson demonstrated increased retropalatal collapse during the expiratory phase in adults with mild obstructive sleep apnea and snoring. ${ }^{8}$ Further understanding and definition of these expiratory related events are required, both in children with evidence of sleep disordered breathing and in normal children.

Here we describe the respiratory, cardiac, and sleep-state characteristics of two types of prolonged sleep-related respiratory pauses during exhalation: post-sigh central apnea (PSCA) and prolonged expiratory apnea (PEA). Both events are suggestive of airflow limitation during exhalation. An augmented breath, which is a sigh, precedes both PEA and PSCA. Both events fulfill criteria for apnea according to standard classifications. These events may be inappropriately categorized as pathologic events when in fact they appear, in this study, to be benign. We hypoth- 
esize that there are changes in autonomic and mechanically mediated physiologic parameters that differentiate the two events. Both events demonstrate a significant decrease in heart rate. The decrease in heart rate is exaggerated in PEA in response to occlusion of the upper airway during exhalation.

\section{METHODS}

This retrospective study evaluated the characteristics of two types of prolonged sleep related respiratory pauses that occur in children. We reviewed all archived outpatient polysomnograms completed on patients aged 5 to 14 years old for routine clinical care between January 2010 and April 2011. All children underwent a comprehensive history and physical examination by a board certified pediatric sleep medicine physician or underwent a comprehensive review of the history and physical exam according to accepted protocol prior to polysomnography. A standardized validated comprehensive sleep history questionnaire (Children's Memorial Pediatric Sleep Questionnaire) was also reviewed by the pediatric sleep medicine specialist. All data were obtained before the polysomnogram. Patients were excluded from analysis if their polysomnogram demonstrated severe obstructive sleep disordered breathing, had developmental delay with static encephalopathy, required supplemental oxygen at baseline, or had significant upper airway abnormalities (Pierre-Robin Sequence, history of tracheostomy, history of laryngotracheal reconstruction, etc.), or if they were unable to complete the study. Studies were not included if there was insufficient data recording for analysis. Studies that were completed after adenotonsillectomy were not included. Studies that met the above inclusion criteria were then screened for the presence of augmented breaths associated with respiratory pauses, described in detail below. Seventeen patients, 5 to 14 years old, had events and complete recording data available for data analysis. Fifty-seven events were included for analysis. This study was approved by the Institutional Review Board of Children's Memorial Hospital.

Comprehensive video-polysomnography was performed on all patients. Standard clinical consent was obtained prior to each polysomnogram. All polysomnograms were obtained on a 32-channel Cadwell Easy III PSG machine (Cadwell Laboratories Inc, Kennewick, WA, USA). Identical techniques and recording montage were used for all patients and consisted of: electroencephalogram ([EEG]: 8 channels), electro-oculography ([EOG]: consisting of left outer canthus and right outer canthus), chin muscle electromyography (EMG), modified lead II electrocardiogram (ECG), and left and right anterior tibialis EMG. Respiratory effort was monitored with inductive plethysmography with separate chest and abdominal belts. Intercostal EMG recording was obtained by placement of an electrode in the $5^{\text {th }}$ intercostal space in the anterior axillary line. Airflow was monitored by nasal pressure transduction, nasal thermistry, and continuous monitoring of the capnography wave form (at the nose and mouth) with breath-to-breath analysis and 10-sec average (Smiths BCI Capnocheck Plus, Smiths Medical, St. Paul, MN, USA). A 3-sec sampling delay occurs with use of a splitlumen cannula. Such a delay was incorporated into scoring of events. Continuous pulse oximetry was recorded for the duration of each study (Cadwell Laboratories Inc, Kennewick, WA,
USA, Nonin 6000ci Sensor, Nonin, Plymouth, MN, USA). All studies included digital video recording. Prior to each study, calibration and graphic representation of inspiration and expiration were validated using polysomnographic methods modified to verify polarity of graphic representation. Inspiration was identified polysomnographically by documenting negative voltage change (producing an upward movement) from an isoelectric baseline for airflow, chest effort, and abdominal effort during prolonged voluntary inspiration. Expiration was identified by documenting positive voltage change (producing downward movement) from an isoelectric baseline for airflow, chest effort, and abdominal effort during prolonged voluntary expiration. All records were scored for sleep staging by an accredited clinical polysomnographer according to the accepted criteria of the AASM 2007 Scoring Manual. $^{2}$

All polysomnograms that met initial inclusion criteria were screened for the presence of augmented breaths. Augmented breaths were defined as an inspiratory/expiratory cycle, (as measured by polarity and voltage change on both chest and abdominal channels) at least $50 \%$ greater than the average cyclic voltage variation for the preceding 4 breaths. Only augmented breaths followed by a respiratory pause $\geq 6 \mathrm{sec}$ were included for analysis. Augmented breaths were accompanied by brief $(<$ $3 \mathrm{sec}$ ) electrocortical EEG arousals. Those augmented breaths associated with major body movements were excluded from analysis. The respiratory events were not included if they had previously been scored as a central, obstructive, or mixed apnea, even if they met the criteria described above. Only events in NREM sleep were included.

All augmented breaths that occurred during sleep were tagged, printed without patient identifiers, and provided to one of the authors (SS) who was blinded on the type of event for classification as a PEA or PSCA. Capnography was used to differentiate the two events. A dual-lumen capnography cannula was used to record changes in $\mathrm{CO}_{2}$ at the nose and mouth. Use of such a device permits accurate capnography recording irrespective of the route of inhalation and exhalation. PEA demonstrates a significantly prolonged alveolar plateau in the capnography wave form after the augmented breath. PSCA may demonstrate a prolonged alveolar plateau, but is proportional to the change in tidal volume with the augmented breath, although not as prolonged as seen in PEA. Additionally, classification was based on the polarity of voltage change on the airflow channel for the first breath following the respiratory pause. Events were classified according to whether the first post-apnea breath was inspiratory or expiratory. When the first breath after the augmented breath and respiratory pause was inspiratory, the event was classified as a PSCA. When the first breath after the augmented breath and associated respiratory pause was expiratory, the event was classified as a PEA. There are some cases in which each event did not hold true to this rule, demonstrating the importance of the role of capnography in identifying these events. Representative images are included and discussed in the results section. Scoring of the events was done in an encrypted manner so as not to bias data analysis.

Cardiac and respiratory variables were calculated and coded by a research fellow, who was blinded to the categorization of each event. Average heart rate and respiratory rate were determined from the average heart rate and respiratory rate for $10 \mathrm{sec}$ 
before the apnea and $10 \mathrm{sec}$ after the tagged events. R-R intervals on ECG were measured from the onset of the augmented breath (beginning at zero baseline crossing) and throughout the apnea with the use of integrated tools within the polysomnogram software. Apnea length and oxygen saturation recorded during and after the events were noted.

All data were entered into a commercially available data base computer program and analyzed using the R system for statistical analysis version 2.12.2 (R Development Core Team). Descriptive analysis was completed on the patient characteristics, results of the polysomnograms, and for the cardiopulmonary variables. Paired comparisons between pre-event vital signs and those during the event were compared using the sign test. Comparisons between the two types of events were performed using the Mann-Whitney U test. The study was powered to detect a $15 \%$ difference between groups with a power of $90 \%$ and a type I error of 0.05 . A $15 \%$ difference in heart rate was determined to be clinically significant based on recently published normative data. ${ }^{9}$ Results are reported as medians and interquartile ranges unless otherwise specified.

\section{RESULTS}

Seventeen patients, 9 girls and 8 boys, were included in the analysis, with an average age of $8.5 \pm 3.5$ years. Indications for referral for polysomnography are listed in Table 1. Sleep architecture and continuity variables are presented in Table $\mathbf{2}$. Fifty-seven augmented breaths followed by apneas $\geq 6 \mathrm{sec}$ were identified. Comparative data for each event, PEA $(n=28)$ and PSCA $(n=29)$, may be found in Table 3. Representative examples of a PEA and PSCA are demonstrated in Figures 1-4. During the augmented breath, all 57 events were associated

Table 1-Summary of referral characteristics

$\begin{array}{lc}\text { Referral Characteristic } & \text { Number of Patients } \\ \text { Snoring } & 14 \\ \text { Restless sleep } & 8 \\ \text { Tonsillar hypertrophy } & 6 \\ \text { Behavioral disturbance } & 4 \\ \text { Sleepiness } & 2 \\ \text { Apnea } & 1 \\ \text { Mouth breathing } & 2 \\ \text { Limb movements in sleep } & 2 \\ \text { Other (allergic rhinitis, asthma, teeth } & 5 \\ \text { grinding, sleep talking, enuresis, obesity) } & \end{array}$

with an electrocortical arousal of 3-4 seconds. The duration of the PEA and PSCA were not significantly different, lasting 10.3 (9.14-12.59) seconds and $9.55(8.4-11.8)$ seconds, respectively $(\mathrm{p}=0.26)$.

Both PEA and PSCA demonstrated a significant difference between the average heart rate during the augmented breath prior to the pause and the lowest instantaneous heart rate during the first half of the pause. In the PEA group, the heart rate dropped from $81.8(76.4-96.4)$ beats per minute to 63.9 (59.41-68.3) beats per minute ( $<<0.001)$. In the PSCA group, the heart rate dropped from 86.85 (73.6-101.45) beats per minute to $66.75(61.7-80.75)$ beats per minute $(p<0.001)$. There was no difference between the lowest heart rate in the first half and second half of the event within each group. A significant difference was noted between the lowest instantaneous heart rate in the second half of each event to the average heart rate after each event $(p<0.001)$. In summary, the heart rate demonstrates a significant decrease from baseline during the initial portion of the respiratory event that is maintained until the end of the apnea, when the heart rate returns to baseline.

When the events were compared to each other, there was statistically significant evidence to discriminate between the

Table 2-Summary of polysomnography results

$\begin{array}{lcc} & \text { Median } & \text { Interquartile Range } \\ \text { Total sleep time(min) } & 367.5 & 329.5-403 \\ \text { Sleep latency (min) } & 26 & 17.5-43.5 \\ \text { REM latency (min) } & 147.3 & 114.2-171.4 \\ \text { Sleep efficiency (\%) } & 77 & 72-89 \\ \text { Stage } \mathrm{N} 1 \% & 3 & 2-3 \\ \text { Stage } \mathrm{N} 2 \% & 48 & 44-50 \\ \text { Stage } \mathrm{N} 3 \% & 33 & 29-36 \\ \text { Stage R\% } & 15 & 11-20 \\ \text { Arousal index } & 16 & 14-21 \\ \text { AHI } & 2 & 1-3 \\ \text { RDI } & 2 & 1-6 \\ \text { REM index } & 2 & 0-6 \\ \text { Hypopneas } & 5 & 2-10 \\ \text { Obstructive apnea } & 0 & 0-1 \\ \text { Mixed apneas } & 0 & - \\ \text { Average } \mathrm{SpO}_{2}(\%) & 97 & 96-98 \\ \text { Low } \mathrm{SpO}_{2}(\%) & 92 & 90-94 \\ \text { Average } \mathrm{EtCO}_{2} & 42.4 & 40.6-44 \\ \text { Max EtCO } & 50 & 49-51 \\ \% \text { Time } \mathrm{EtCO}_{2}>50 & 0 & 0-0.2\end{array}$

Table 3-Summary data for PEA and PSCA (median and interquartile range)

PEA

Event duration (s)
HR prior (bpm)
Lowest $1^{\text {st }}$ half HR (bpm)
Lowest $2^{\text {nd }}$ half HR (bpm)
HR after (bpm)

$10.3(9.14-12.59)$
$81.8(76.4-96.4)$
$63.9(59.41-68.3)^{\star}$
$66.08(60.0-68.5)$
$82.4(76.2-89.9)^{\ddagger}$

PSCA

$9.55(8.4-11.8)$
$86.85(73.6-101.45)$
$66.75(61.7-80.75)^{\star}$
$66.08(58.68-78.63)$
$87.2(73.05-97.47)^{\ddagger}$

p-Value Mann Whitney $\mathbf{U}^{+}$
0.26
0.678
0.03
0.38
0.79

${ }^{*}(p<0.001)$ for HR comparison prior to and during first half for PEA and PSCA, respectively. ${ }^{\ddagger}(p<0.001)$ for HR comparison during second half of the event and after the respiratory event for PEA and PSCA, respectively. ${ }^{+}$Comparison between PEA and PSCA. 
Figure 1-Prolonged expiratory apnea, 30-sec epoch

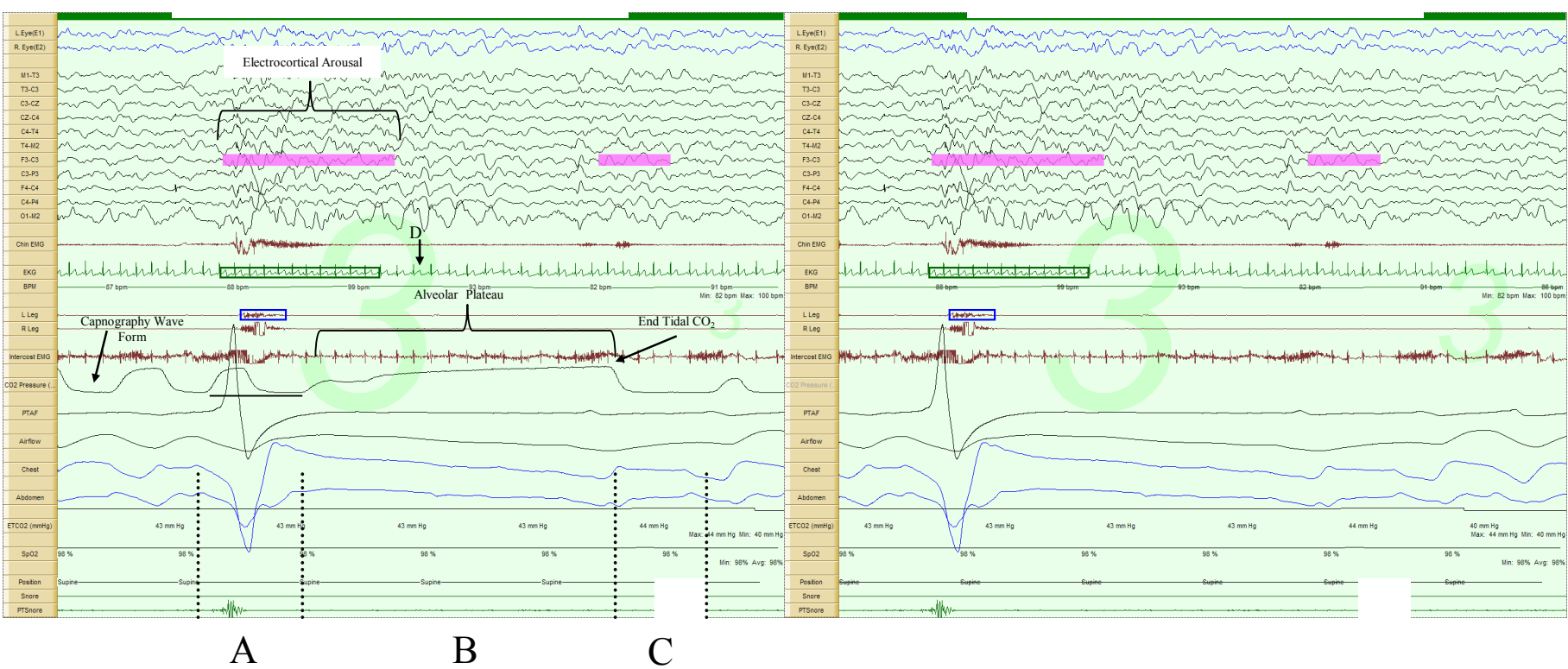

The image on the left includes the capnography tracing, while the one on the right does not. Augmented breath (A); respiratory pause (B); first post-pause breath $(C)$ in the direction of exhalation (positive polarity); lowest instantaneous heart rate during the respiratory pause (D). Note the prolonged alveolar plateau phase in the capnopgraphy tracing. Note there is about a 3- to 5-sec sampling delay in the capnography tracing, as illustrated by the horizontal line below the tracing.

Figure 2-Prolonged expiratory apnea, 60-sec epoch

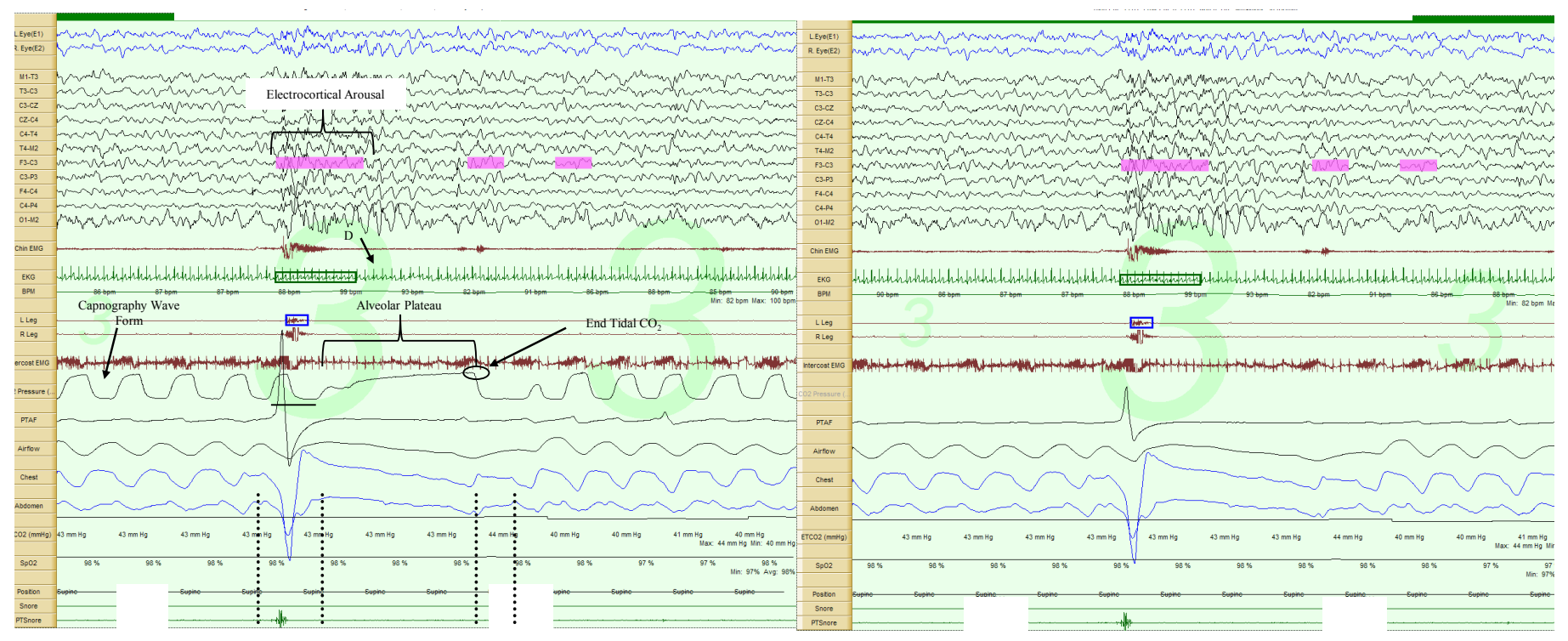
A
B
$\mathrm{C}$

The image on the left includes the capnography tracing, while the one on the right does not. Augmented breath $(\mathbf{A})$; respiratory pause (B); first post-pause breath $(\mathbf{C})$ in the direction of exhalation (positive polarity); lowest instantaneous heart rate during the respiratory pause (D). Note the prolonged alveolar plateau phase in the capnopgraphy tracing. Note there is about a 3- to 5-sec sampling delay in the capnography tracing, as illustrated by the horizontal line below the tracing.

two episodes. Specifically, the lowest instantaneous heart rate recorded in the first half of each event was statistically significantly lower in the PEA group when compared to the PSCA group (63.9 [59.41-68.3] vs 66.75 [61.7-80.75] beats per minutes, $p=0.03$ ). However, the percentage decrease in heart rate is statistically different. The heart rate in
PEA decreases a median $23.8 \%$ (IQR: $17.2 \%$ to $32.2 \%$ ). The heart rate in PSCA decreases a median 18.1\% (IQR: 14.1\% to $22.8 \%$ ) (Mann-Whitney U Test $p=0.03$ ). No significant difference was seen comparing the lowest instantaneous heart rate in the second half of the PEA group and PSCA group $(\mathrm{p}=0.38)$. There was no statistical significance between the 
Figure 3-Post-sigh central apnea, 30-sec epoch

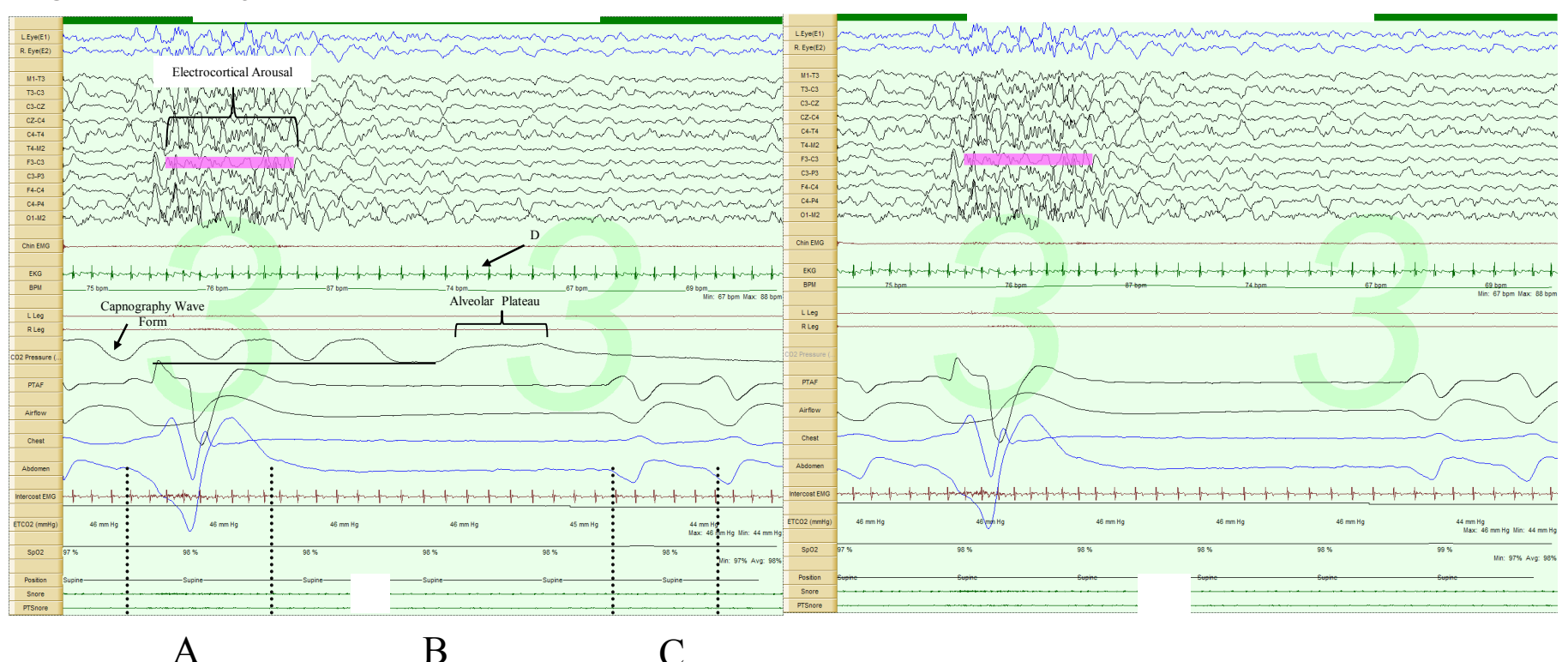

The image on the left includes the capnography tracing, while the one on the right does not. Note the augmented breath $(\mathrm{A})$ and respiratory pause $(\mathrm{B})$. The first post-pause breath $(C)$ is in the direction of inspiration (negative polarity); lowest instantaneous heart rate during the respiratory pause (D). Note there is about a 3- to 5-sec sampling delay in the capnography tracing, as illustrated by the horizontal line below the tracing.

Figure 4-Post-sigh central apnea, 60-sec epoch
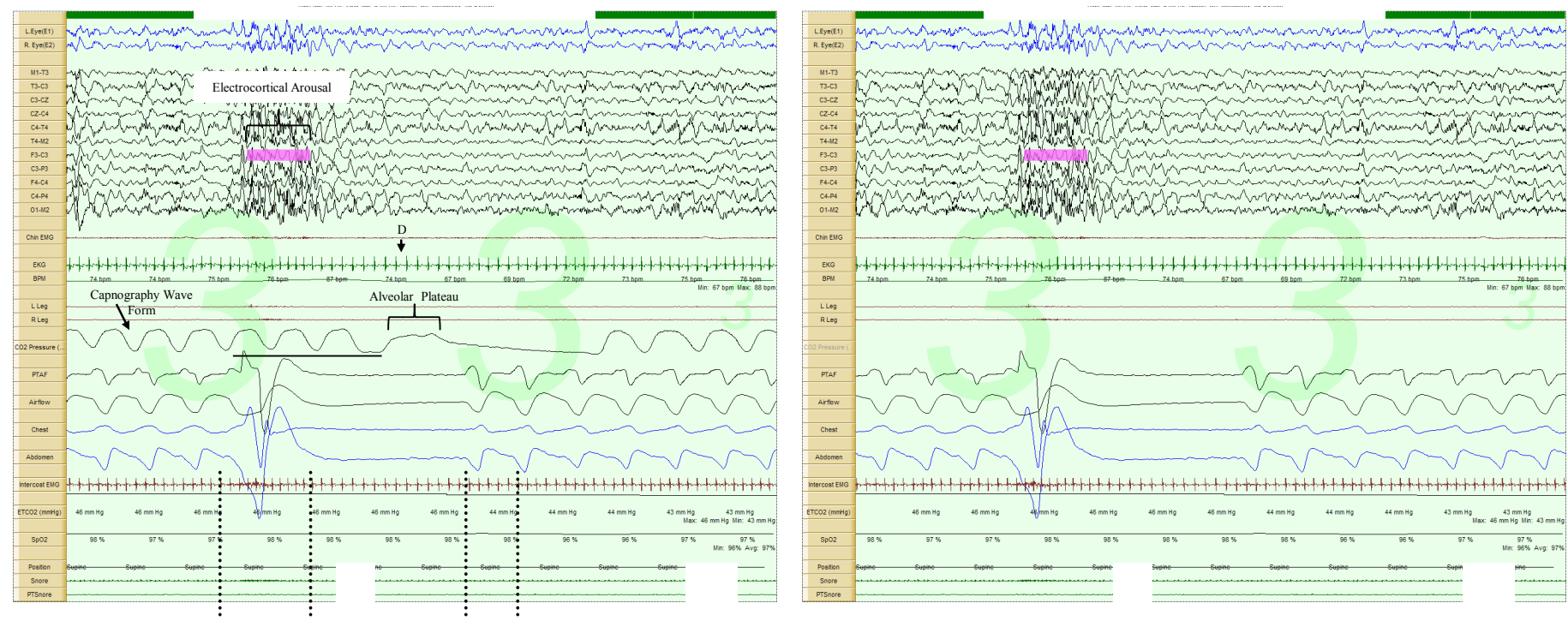

The image on the left includes the capnography tracing, while the one on the right does not. Note the augmented breath $(\mathrm{A})$ and respiratory pause $(\mathrm{B})$. The first post-pause breath $(C)$ is in the direction of inspiration (negative polarity); lowest instantaneous heart rate during the respiratory pause (D). Note there is about a 3- to 5-sec sampling delay in the capnography tracing, as illustrated by the horizontal line below the tracing.

heart rate prior to or after each event, suggesting that the difference in heart rates was not due to a baseline difference in heart rate. There was no significant difference in age between the two groups $(p=0.96)$.

Interestingly, during split screen video/audio recording, PEA was associated with continuous expiratory stridulous vocalizations, audible expiratory braking, and/or visible forced expiratory effort.

\section{DISCUSSION}

Although brief central apneas during active/REM sleep are common and are considered normal physiological occurrences, prolonged central apneas (especially during quiet/NREM sleep) may be classified as pathological if lasting longer than $20 \mathrm{sec}-$ onds, or if associated with oxygen desaturations or bradycardia. Typically, three types of apnea are described in infants and chil- 
dren: obstructive, central and mixed. These events are based on physiologic changes associated with the inspiratory portion of the respiratory cycles. However, significant respiratory disturbances occur during exhalation as well. In 1982, Onal observed prolongation of the expiratory phase of the respiratory cycle during NREM sleep central apneas. ${ }^{10}$ Southall described prolonged forced expiration against obstruction of the upper airway resulting in cyanotic spells in infants. ${ }^{3}$ Vocal cord adduction and glottic closure have been identified in children who have suffered apparent life-threatening events (ALTEs) and during the central pause associated with periodic breathing. ${ }^{11}$ Perez-Padilla and Issa describe the characteristics of sighs during sleep in adults, presenting a similar description to PSCA. ${ }^{12,13}$ Eiselt describes the role of sighs in the respiration of preterm and term infants, specifically focusing on lung volume recruitment and associated heart rate changes. ${ }^{14}$ These studies demonstrate that respiratory events during expiration and sighs occur during sleep. There is little literature specifically describing these events and their role in the spectrum of upper airway resistance syndromes and sleep disordered breathing in children. PSCA and PEA are unique expiratory related respiratory events and no literature describes them.

We described two types of respiratory events that may appear similar to pathological central apnea in infants and children when limited technology is used, but are associated with continued expiratory phase of the respiratory cycle, as demonstrated by the prolonged alveolar plateau noted during capnography. When reviewed without the capnography tracing, all PSCA and PEA included in this analysis could be scored as central apnea based on 2007 AASM criteria. The prolonged alveolar plateau noted in the PEA, however, is suggestive of continued airflow during expiration, despite what appears to be an apneic event based on lack of abdominal and chest movement. Capnography can identify variations in respiratory patterns in children, and we feel this is a key component of defining these events. ${ }^{15}$ Both PSCA and PEA follow an augmented inspiration and are of sufficient duration to meet the current definition of pathological central apnea in children (PSCA range 6.54 to 14.1 seconds, PEA 6.8 to 15.2 seconds). PEA was associated with audible expiratory braking or expiratory stridor during the immediate post-inspiratory phase. Statistically significant changes in heart rate were identified when PSCA events were compared with PEA events. Lowest instantaneous heart rate occurred during a PEA and reached a nadir of 36 beats per minute in one case. A nadir to this degree would typically occur within the range of bradycardia considered pathological on pneumography and/or event recordings, but did not appear to be associated with any untoward events during these studies. In a central apnea, the heart rate nadir occurs in the second half of the event and results in an arousal. In PSCA and PEA, the heart rate nadir occurs in the first half of the event. PSCA and PEA are preceded by an arousal.

PSCA and PEA differ from pathological central apnea in several important ways. First, PSCA and PEA are preceded by an augmented breath and brief ( $<3$ second) electrocortical arousal noted on EEG. Pathological central apneas are typically not preceded by an augmented breath or arousal. Instantaneous heart rate reached its nadir immediately after augmented breaths and gradually returned to baseline during the last half of the respiratory pause during a PEA. Similar changes in heart are seen during inspiratory Valsalva maneuver. ${ }^{16}$ The pattern of heart rate changes in PSCA and PEA is similar. However, it is clear that the heart rate decreases further in PEA. This may reflect narrowing of the upper airway during exhalation resulting in a Valsalva-like maneuver in PEA events that is not seen in PSCA events. Pathological central (as well as obstructive) apnea is typically associated with a gradual deceleration in heart rate, with the nadir of instantaneous heart rate occurring during the last third of the respiratory pause, immediately prior to the arousal. The bradycardia is a result of a vagal response to decreased cardiac preload in the setting of absent respiratory motion of the chest. Finally, despite the prolonged nature of these respiratory pauses, they appeared, in our sample, to be associated with minimal physiological consequences. Oxygen saturation rarely fell more than 3\% from baseline (data not shown). Pathological apneas are often associated with gradual oxygen desaturation as the respiratory pause continues.

Interestingly, PEA events were often associated with continuous expiratory vocalizations and/or visible forced expiratory effort. Given the combination of expiratory vocalization and a prolonged expiratory pause preceded by an augmented breath, one would be correct to make a possible association between the events we describe and catathrenia. Catathrenia is defined as a chronic sleep disorder characterized by expiratory groaning during sleep. According to the International Classification of Sleep Disorders, $2^{\text {nd }}$ edition (ICSD-2), catathrenia is identified during polysomnography as a deep inhalation followed by a prolonged exhalation during which moaning noises are produced and may last between 2 and 49 seconds, with an increased frequency during the late REM cycles. ${ }^{17}$ Recent studies have demonstrated that catathrenia, or catathrenia-like symptoms, can be successfully treated with continuous positive airway pressure (CPAP) in adult patients. ${ }^{18}$ This suggests that catathrenia results from upper airway resistance and occlusion during exhalation. The origin of the sounds during catathrenia is different from inspiratory snoring, again suggesting a different mechanism, namely upper airway resistance. ${ }^{19}$ Catathrenia vocalizations occur during REM sleep and tend to occur in clusters. All of the events we demonstrate here occurred during NREM sleep and were isolated occurrences without evidence of a temporal pattern.

Clinical significance of PSCA and PEA is unknown and may be an age-related phenomenon in the pediatric population, resulting in more severe consequences in infants but not in older children. However, these events merit attention, as they may result in incorrect diagnoses and inappropriate evaluations based on misinterpretation or misclassification of the event.

Our study has limitations, specifically the true incidence of expiratory apnea events in children is not known. Selection bias is present in this study, given that the polysomnograms used for analysis were done for children with suspected sleep disordered breathing, not for otherwise normal children. We attempted to limit this effect by selecting studies from patients who did not demonstrate significant sleep disordered breathing or major upper airway abnormalities. Determination of age dependence and incidence of abnormal expiratory events in children analysis of polysomnograms in normal children for these specific events is needed. 
In summary, we describe two unique expiratory related respiratory events that occur during sleep in children. PEA and PSCA demonstrate stereotypic heart rate changes and may resemble pathologic apneas. Both PEA and PSCA can be identified by an augmented breath followed by a prolonged respiratory pause. The heart rate significantly decreases from baseline during both events, more so in PEA, before returning to baseline. The longterm sequelae and clinical significance of these events is yet to be identified and understood. Future prospective studies are required to determine the frequency of these events in children with and without sleep disordered breathing. Given the similar appearance of these events to pathologic apneas and the potential for unnecessary evaluation, addition of PEA and PSCA to polysomnographic scoring manuals should be considered.

\section{ABBREVIATIONS}

PEA, prolonged expiratory apnea

PSCA, post-sigh central apnea

CPAP, continuous positive airway pressure

EEG, electroencephalogram

EOG, electro-oculography

EMG, electromyography

ECG, electrocardiogram

\section{REFERENCES}

1. Haddad GG, Farber JP. Developmental neurobiology of breathing. New York: Dekker; 1991.

2. Iber C, Ancoli-lsrael S, Chesson A, Quan SF for the American Academy of Sleep Medicine. The AASM manual for the scoring of sleep and associated events: rules, terminology and technical specifications, 1st ed.: Westchester, IL: American Academy of Sleep Medicine, 2007.

3. Southall DP, Talbert DG, Johnson P, et al. Prolonged expiratory apnoea: a disorder resulting in episodes of severe arterial hypoxaemia in infants and young children. Lancet 1985;2:571-7.

4. Series F, Marc I. Accuracy of breath-by-breath analysis of flow-volume loop in identifying sleep-induced flow-limited breathing cycles in sleep apnoea-hypopnoea syndrome. Clin Sci (Lond) 1995;88:707-12.

5. Stanescu D, Kostianev S, Sanna A, Liistro G, Veriter C. Expiratory flow limitation during sleep in heavy snorers and obstructive sleep apnoea patients. Eur Respir J 1996;9:2116-21.

6. Cochrane DD, Adderley R, White CP, Norman M, Steinbok P. Apnea in patients with myelomeningocele. Pediatr Neurosurg 1990;16:232-9.
7. Stevenson KL. Chiari Type II malformation: past, present, and future. Neurosurg Focus 2004;16:E5.

8. Woodson BT. Expiratory pharyngeal airway obstruction during sleep: a multiple element model. Laryngoscope 2003;113:1450-9.

9. Flemming S, Thompson M, Stevens R. Normal ranges of heart rate and respiratory rate in children from birth to 18 years of age: a systematic review of observational studies. Lancet 2011:377:1011-8.

10. Onal E, Lopata M, O'Connor T. Pathogenesis of apneas in hypersomnia-sleep apnea syndrome. Am Rev Respir Dis 1982;125:167-74.

11. Ruggins NR, Milner AD. Site of upper airway obstruction in infants following an acute life-threatening event. Pediatrics 1993;91:595-601.

12. Perez-Padilla R, West $P$, Kryger M. Sighs during sleep in adult humans. Sleep 1983;6:234-43.

13. Issa FG, Porostocky $\mathrm{S}$. Effect of sleep on changes in breathing pattern accompanying sigh breaths. Respir Phsyiol 1993:175-87.

14. Eiselt M, Curzi-Dascalova L, Leffler $C$, et al. Sigh-related heart rate changes during sleep in premature and full-term newborns. Neuropediatrics 1992;23:286-91.

15. Tirosh E, Bilker A, Bader D, Cohen A. Capnography in spontaneously breathing preterm and term infants. Clin Physiol 2001;21:150-4.

16. Looga R. The Valsalva manoeuvre--cardiovascular effects and performance technique: a critical review. Respir Physiol Neurobiol 2005;147:39-49.

17. American Academy of Sleep Medicine. The international classification of sleep disorders: diagnostic and coding manual. 2nd ed. Westchester, IL: American Academy of Sleep Medicine; 2005.

18. Abbasi AA, Morgenthaler TI, Slocumb NL, Tippmann-Peikert M, Olson EJ, Ramar K. Nocturnal moaning and groaning-catathrenia or nocturnal vocalizations. Sleep Breath 2012;16:367-73.

19. Iriarte J, Fernandez S, Fernandez-Arrechea N, et al. Sound analysis of catathrenia: a vocal expiratory sound. Sleep Breath 2011;15:229-35.

\section{ACKNOWLEDGMENTS}

Work for this study was performed at Children's Memorial Hospital, Chicago IL.

\section{SUBMISSION \& CORRESPONDENCE INFORMATION}

Submitted for publication November, 2011

Submitted in final revised form April, 2012

Accepted for publication April, 2012

Address correspondence to: Mark Haupt, M.D., Ann \& Robert H. Lurie Children's Hospital of Chicago, Division of Pulmonary Medicine, Box \#43, 225 East Chicago Ave., Chicago, IL 60610; Tel: (312) 227-6260; Fax: (312) 227-9419; E-mail: mhaupt@ luriechildrens.org

\section{DISCLOSURE STATEMENT}

This was not an industry supported study. The author has indicated no financial conflicts of interest. 